\title{
Numerical Linked-Cluster Algorithms. I. Spin systems on square, triangular, and kagomé lattices
}

\author{
Marcos Rigol \\ Department of Physics and Astronomy, University of Southern California, Los Angeles, California 90089, USA \\ Tyler Bryant and Rajiv R. P. Singh \\ Department of Physics, University of California, Davis, California 95616, USA
}

(Dated: October 25, 2018)

\begin{abstract}
We discuss recently introduced numerical linked-cluster (NLC) algorithms that allow one to obtain temperature-dependent properties of quantum lattice models, in the thermodynamic limit, from exact diagonalization of finite clusters. We present studies of thermodynamic observables for spin models on square, triangular, and kagomé lattices. Results for several choices of clusters and extrapolations methods, that accelerate the convergence of NLC, are presented. We also include a comparison of NLC results with those obtained from exact analytical expressions (where available), high-temperature expansions (HTE), exact diagonalization (ED) of finite periodic systems, and quantum Monte Carlo simulations. For many models and properties NLC results are substantially more accurate than HTE and ED.

PACS numbers: 05.50.+q,05.70.-a,75.10.Jm,05.10.-a
\end{abstract}

\section{INTRODUCTION}

Accurate quantitative calculations of finitetemperature properties of quantum lattice models are a challenging task [1, 2]. One of the few general methods that works directly in the thermodynamic limit, is that of high-temperature expansions (HTEs), where properties of the system are expanded in powers of inverse temperature, $\beta=1 / T$ [3] . These expansions, carried out to order $\beta^{N}$ (where $N$ is typically around 10 ), provide highly accurate numerical results at high temperatures. However, below some temperature $T_{s}$ related to the relevant microscopic energy scale of the system, the series diverges. Such a divergence need not be related to any finite-temperature phase transitions or long-range correlations. For example, in low dimensional or frustrated spin systems, often there is either no finite-temperature phase transition or such a transition occurs well below the exchange constant $J$. The microscopic energy scale $J$ still controls the radius of convergence of the high-temperature series.

Beyond the radius of convergence $\beta>\beta_{s}$, series extrapolation methods [4] allow one to calculate thermodynamic properties, but their reliability remains uncertain. Our motivation for developing the numerical linked cluster (NLC) method is to be able to obtain the properties of these systems, in the thermodynamic limit, for $\beta>\beta_{s}$ in a more reliable way, especially if the correlations in the system remain short ranged. NLC uses the linked cluster basis of HTE, but replaces the expansion in $\beta$ by an exact numerical calculation of the properties of linked clusters at any temperature [5, 6]. In any practical implementation of NLC, only the contributions from clusters up to some maximum size are included. This can lead to highly accurate properties of the thermodynamic system, even beyond the radius of convergence of HTE, provided the correlations are short ranged. Thus, NLC helps to sepa- rate cases where the failure of HTE is due to its analytic structure in the complex plane, from where the correlations truly exceed the largest clusters studied. We would like to emphasize that this does not imply that comparable results for thermodynamic systems can be obtained simply by exact diagonalization (ED) of individual periodic clusters of size comparable to the maximum size used in NLC. We will show that NLC can be substantially more accurate than ED for finite-temperature properties of a thermodynamic system. Furthermore, one can accelerate the convergence of NLC, even when correlation length exceeds the largest cluster studied, by using sequence extrapolation techniques, which are in many ways analogous to series extrapolation methods [4, 7].

We present in this paper a detailed exposition of the NLC algorithm. The basic method was already presented in Ref. [5]. Here we discuss in detail the different choices of clusters that can be used to build the numerical expansion. We also detail different extrapolation methods that, like Pade approximants for HTE, allow one to accelerate the convergence of NLC. These methods are especially relevant for the application of NLC to models in which correlations build up rapidly with reduction in temperature. Comparisons between results obtained by means of NLC with known techniques such as exact diagonalization (ED), quantum Monte Carlo (QMC) simulations, and HTE, are also presented.

The exposition is organized as follows. In Sec. III, we introduce the basis of NLC. We then present (Sec. III) an overview of different sequence extrapolation methods that can help accelerate the convergence of NLC. The rest of the manuscript is devoted to show how to build the series for spin models on square (Sec. IV), triangular (Sec.VI), and kagomé (Sec. VI) lattices. Different choices of clusters are discussed in detail, and applied to Ising, $X Y$, and Heisenberg models. Finally, the conclusions are presented in Sec. VII. 


\section{BASIS OF NLC}

The fundamental basis for a linked cluster expansion, for some extensive property $P$ of an infinite lattice $\mathcal{L}$, is the relation [3, 8]

$$
P(\mathcal{L}) / N=\sum_{c} L(c) \times W_{P}(c),
$$

where the left hand side is the value of the property $P$ per lattice site in the thermodynamic limit. On the righthand side $L(c)$ is the so-called lattice constant, which is the number of embeddings of the cluster $c$, per lattice site, in the lattice $\mathcal{L}$ (explicit examples will be given later). $W_{P}(c)$ is the weight of the cluster $c$ for the property $P$. The latter is defined recursively by the principle of inclusion and exclusion [3],

$$
W_{P}(c)=P(c)-\sum_{s \subset c} W_{P}(s),
$$

where $P(c)$ is the property $P$ calculated for the finite cluster $c$. The sum on $s$ runs over all subclusters of $c$. In HTE, for every cluster, $P(c)$ and hence its weight $W_{P}(c)$ are expanded in powers of $\beta$ and only a finite number of terms are retained. In NLC an exact diagonalization of the cluster is used to calculate $P(c)$ and $W_{P}(c)$ at any temperature. [Notice that in contrast to the clusters used in ED studies, the ones in Eq. (2) do not have periodic boundary conditions.] The exact numerical calculation of $P(c)$ allows NLC to build more bare information of the system than HTE.

There is another aspect in which the NLC scheme is fundamentally different from HTE, and that can be used to ones advantage. In HTE, the choice of clusters is dictated solely by the need to get the power series expansion in $\beta$ to as high an order as possible. This typically means that clusters are defined and ordered by the number of bonds. In NLC, one has substantial freedom to select the set of clusters and the order in which they are considered. One can arrange them by number of sites, by number of bonds or, as we will see below, one can even consider a reduced set of clusters. In order to obtain correct results at high temperatures, one requirement is that with increasing order, the cluster weights, when expanded in inverse temperature, should give the correct HTE coefficients as well. This ensures that when HTE converges, NLC gives results that are identical to HTE. However, as we will see, NLC expansions may involve a choice of clusters that sacrifice efficiency in the order to which they give the exact HTE coefficients, for better results at intermediate and low temperatures.

As discussed before, when correlations are shorter ranged than the size of clusters that are included in any implementation of NLC, the NLC results are accurate without any need for extrapolations. On the other hand, for systems with an ordered ground state, such an implementation must eventually break down as the temperature is lowered (larger clusters will start making large contributions). In the next section we discuss some "tricks" that can be used to accelerate the convergence of the direct sum in Eq. (1). This can lead to accurate thermodynamic results at temperatures where correlation length far exceeds the sizes of the cluster included in the sum.

\section{SEQUENCE EXTRAPOLATIONS}

In this section, we consider the general problem of estimating $P(\mathcal{L})$ in Eq. (11), when the weights $W_{P}$ are only known for clusters up to a given size. In order to produce a sensible extrapolation scheme one can group clusters together and define

$$
S_{n}=\sum_{c} L\left(c_{n}\right) \times W_{P}\left(c_{n}\right),
$$

where all clusters $c_{n}$ share a given characteristic, which could be, for example, the number of bonds, sites, etc. Equation (11) can be rewritten as

$$
P(\mathcal{L})=\sum_{n} S_{n}
$$

and one can define partial sums as

$$
P_{n}(\mathcal{L})=\sum_{i=1}^{n} S_{i}
$$

So, our goal is to estimate $P(\mathcal{L})=\lim _{n \rightarrow \infty} P_{n}$ from a sequence $\left\{P_{n}\right\}$, which is known for $n=1, \ldots, N$.

Several methods have been developed to accelerate the convergence of such sequences. An extensive review with references to original papers can be found in Ref. [4], and they are similar to series extrapolation methods. We will briefly describe here three methods that we have implemented, and that have proven to be particularly useful in accelerating the convergence of NLC. These methods are known as the Wynn's $(\varepsilon)$ algorithm [4], the Brezinski's $(\theta)$ algorithm [4], and the Euler's transformation 7]. A very important topic that is discussed neither here nor in Refs. [4, 7] is one of error estimation. This is because most studies of errors associated with such extrapolation methods depend on the underlying function satisfying certain properties. As one might expect, these properties, in general, cannot be verified for many of the problems one finds in statistical mechanics.

Wynn's algorithm is defined as follows 37]

$$
\begin{aligned}
\varepsilon_{n}^{(-1)} & =0, \quad \varepsilon_{n}^{(0)}=P_{n}, \\
\varepsilon_{n}^{(k)} & =\varepsilon_{n+1}^{(k-2)}+\frac{1}{\Delta \varepsilon_{n}^{(k-1)}},
\end{aligned}
$$

where the discrete differentiating operator $\Delta$ is only applied to subscripts

$$
\Delta \varepsilon_{n}^{(k-1)}=\varepsilon_{n+1}^{(k-1)}-\varepsilon_{n}^{(k-1)} .
$$


Within this scheme the even entries $\varepsilon_{n}^{(2 k)}$ are expected to converge to $P(\mathcal{L})$, while the odd ones $\varepsilon_{n}^{(2 k+1)}$ diverge. Nonlinear sequence extrapolations usually display this behavior, and it implies that one has to be careful with round-off errors.

One should notice that two iterations are needed for each level of improvement so the new sequence is two terms shorter. We refer to each level of improvement as a cycle. For the problems we have studied so far by means of NLC, Wynn's algorithm has been, in general, the most successful in extending the region of convergency to lower temperatures.

Brezinski's algorithm is defined as follows:

$$
\begin{aligned}
\theta_{n}^{(-1)} & =0, \quad \theta_{n}^{(0)}=P_{n}, \\
\theta_{n}^{(2 k+1)} & =\theta_{n}^{(2 k-1)}+\frac{1}{\Delta \theta_{n}^{(2 k)}}, \\
\theta_{n}^{(2 k+2)} & =\theta_{n+1}^{(2 k)}+\frac{\Delta \theta_{n+1}^{(2 k)} \Delta \theta_{n+1}^{(2 k+1)}}{\Delta^{2} \theta_{n}^{(2 k+1)}},
\end{aligned}
$$

once again the discrete differentiating operator $\Delta$ is only applied to subscripts as in Eq. (7) and

$$
\Delta^{2} \theta_{n}^{(k)}=\theta_{n+2}^{(k)}-2 \theta_{n+1}^{(k)}+\theta_{n}^{(k)} .
$$

As for Wynn's algorithm, only even entries are expected to converge to $P(\mathcal{L})$. Once again, we have a cycle of improvement after two iterations, and for the Brezinski algorithm three terms are lost in each cycle. This fact, together with the second derivative in the denominator in $\theta_{n}^{(2 k+2)}$ [Eq. (8)], reduce the number of cycles one can perform with the Brezinski algorithm as compared to Wynn's algorithm.

Finally, for alternating series, i.e., series in which terms $S_{n}$ [Eq. (4)] alternate in sign $\left[S_{n}=(-1)^{n} u_{n}\right]$, there is a powerful tool known as the Euler transformation [7]. With this algorithm $P_{\infty}(\mathcal{L})$ is approached by the sum $(n$ is even)

$$
u_{0}-u_{1}+u_{2} \ldots-u_{n-1}+\sum_{s} \frac{(-1)^{s}}{2^{s+1}}\left[\Delta^{s} u_{n}\right],
$$

where $\Delta$ is the forward difference operator

$$
\begin{aligned}
\Delta u_{n} & =u_{n+1}-u_{n}, \\
\Delta^{2} u_{n} & =u_{n+2}-2 u_{n+1}+u_{n}, \\
\Delta^{3} u_{n} & =u_{n+3}-3 u_{n+2}+3 u_{n+1}-u_{n}, \ldots .
\end{aligned}
$$

It is always advisable to do the sum of a small number of terms directly, through term $n-1$, and then apply the transformation.

As we will show later, we have found Euler's transformation to be particularly useful for calculations of the specific heat. Having stated that NLC provides a scheme that similar to HTE allows for systematic extrapolations, we should add some remarks here. What the NLC scheme misses is the analytic structure of HTE.
These have proven particularly useful in studies of critical phenomena, where the region of diverging correlation length has also been addressed using Pade extrapolations [4]. We have not yet investigated if NLC can be used to study critical phenomena, as our focus has been on models that do not order down to very low temperatures.

The analytic structure of HTE also allows for changes of variables, and in some cases this can be used in very ingenious ways. For example, recent work by Bernu and Misguich [9], has shown that by converting the expansion for entropy in the inverse-temperature variable to an expansion for entropy in the internal energy, one can develop a powerful extrapolation scheme that can build the ground-state energy and low-temperature power-law behavior into the extrapolation of high-temperature expansions. However, we note that such a scheme is only known for the entropy (and related quantities) and not for susceptibilities or correlation functions. We will show that for the specific heat (and entropy) of two-dimensional Heisenberg antiferromagnets the NLC scheme works better than direct extrapolation of HTE, but the method of Bernu and Misguich is better (at least for square and triangular lattice Heisenberg models) in that it allows estimates all the way down to $T=0$.

In what follows we study thermodynamic quantities (internal energy, entropy, specific heat, and uniform susceptibility) of spin models to show how different clusters and extrapolation techniques can be used to build NLC on square, triangular, and kagomé lattices.

\section{SQUARE LATTICE}

In this section we consider the square lattice. We discuss three different cluster schemes that we have used to build our NLC expansions.

The first, and most natural, choice is to consider all clusters and order them by the number of bonds. This selection has been called "Weak Embeddings" in the series expansion literature [3], and is typically used for HTE. In Fig. 1. we show all clusters that have up to three bonds, and their lattice constant.

Notice that one must include the single site cluster, which corresponds to zero bonds. It dominates observables such as the entropy at very high temperatures. For the smallest bond clusters, such as the one with one bond $(c=2)$ and the first with two bonds $(c=3)$, it is easy to realize that $L(c)=2$ since in the square lattice they can be only placed horizontally, and vertically. The second cluster with two bonds $(c=4)$ can be placed in four ways $[L(c)=4]$, as one can realize rotating it by $90^{\circ}$, and so on.

It is convenient to group together all clusters that have the same Hamiltonian, and diagonalize them just once. Since, the Hamiltonian depends on the topology of how the sites are connected, we call them topological clusters. Looking at our example, clusters $c=3$ and 4 , or $c=6-9$ have the same topology. For calculating thermodynamic 


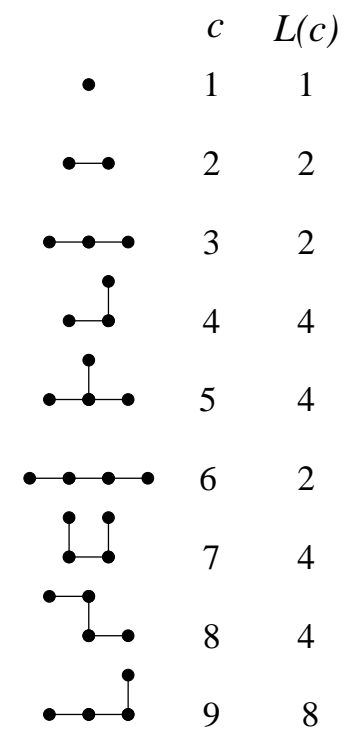

FIG. 1: All clusters with up to three bonds and their lattice constant for the square lattice.

properties, all clusters with the same topology make the same contribution. For the square lattice, we have calculated all possible clusters, and their lattice constants, up to 14 bonds. The number of topological clusters, and sum of $L(c)$, when grouped by their number of bonds is presented in Table. [.

A second choice is to identify clusters by sites. When building the Hamiltonian for such expansion, one places all possible bonds that connect any pair of sites in the graph. This leads to a set of clusters and embeddings that are called "strong embeddings" in the series expansion literature [3], and is typically used for generating the

TABLE I: Number of topological clusters and sum of the lattice constants for clusters with up to 14 bonds in the square lattice. The cluster with 0 bonds is the one site graph.

\begin{tabular}{crr}
\hline \hline No. of bonds No. of topological clusters & $\sum L(c)$ \\
\hline 0 & 1 & 1 \\
1 & 1 & 2 \\
2 & 1 & 6 \\
3 & 2 & 22 \\
4 & 4 & 88 \\
5 & 6 & 372 \\
6 & 14 & 1628 \\
7 & 28 & 7312 \\
8 & 68 & 33466 \\
9 & 156 & 155446 \\
10 & 399 & 730534 \\
11 & 1012 & 3466170 \\
12 & 2732 & 16576874 \\
13 & 7385 & 79810756 \\
14 & 20665 & 386458826 \\
\hline \hline
\end{tabular}

"low-temperature expansions" for Ising models. They can be used to generate HTEs as well. However, different clusters, with a given number of sites, will contribute to HTE in different orders. Thus, the order to which the HTE is correct will be determined by a subset of the clusters with the same number of sites that happen to contribute in the lowest order. On the other hand, having lots of compact clusters, with multiple connectivity between points, could mean that they can give better results beyond the radius of convergence of HTE. In Fig. 2 we show all clusters that have up to four sites, and their lattice constant.

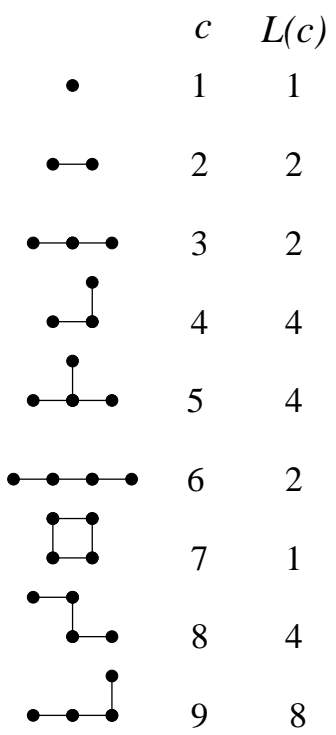

FIG. 2: All clusters with up to four sites and their lattice constant for the square lattice.

By comparing Figs. 1 and 2 one can see that the latter never includes graphs such as $c=7$ of the former one, i.e., all squares are always closed in the site expansion, hence the name "strong embeddings". In addition, while each site in the square lattice has four nearest-neighbor sites, each bond has six nearest-neighbor bonds, which implies that the number of bond clusters increases much faster than the number of site clusters. In the latter case, we have calculated all possible site graphs with up to 16 sites. Their number of topological clusters and sum of lattice constants, when grouped by number of sites, is shown in Table [

Looking at Tables 1 and II one can see that for NLC calculations of bond and site based expansions the main limitation is the computing time (too many clusters) [38], and not the memory as is usual for full diagonalization studies of clusters with periodic boundary conditions. Within NLC one can, however, change that order of limitations considering more complicated (larger) building units for the clusters. Hence, drastically reducing the number of different clusters to consider [5].

A natural selection of a larger building unit in the square lattice is, of course, the elementary plaquette or square. In this case, a consistent NLC scheme requires 
TABLE II: Number of topological clusters and sum of lattice constants for clusters with up to 16 sites in the square lattice.

\begin{tabular}{rrr}
\hline \hline No. of sites No. of topological clusters & $\sum L(c)$ \\
\hline 1 & 1 & 1 \\
2 & 1 & 2 \\
3 & 1 & 6 \\
4 & 3 & 19 \\
5 & 4 & 63 \\
6 & 10 & 216 \\
7 & 19 & 760 \\
8 & 51 & 2725 \\
9 & 112 & 9910 \\
10 & 300 & 36446 \\
11 & 746 & 135268 \\
12 & 2042 & 505861 \\
13 & 5450 & 1903890 \\
14 & 15197 & 7204874 \\
15 & 42192 & 27394666 \\
16 & 119561 & 104592937 \\
\hline \hline
\end{tabular}

that each bond belongs to only one square. This means that we build our clusters out of every alternate square. Different squares can only share sites, which are the zeroth order of the square expansion, and are properly subtracted when calculating the weights in Eq. (2). In Fig. 3 we show all clusters, with up to three such squares, required for a consistent square based NLC expansion.

The calculation of all possible clusters up to five squares (up to 16 sites) is in this case very simple. In Table III we show the results for the number of topological clusters and sum of their lattice constants.

In the next subsections we apply the different NLC expansions detailed above to several well known spin models. All calculations were done on $(3.2 \mathrm{GHz})$ Pentium IV personal computers in times that span between $16 \mathrm{~h}$ for the square based NLC expansion (up to 5 squares) and

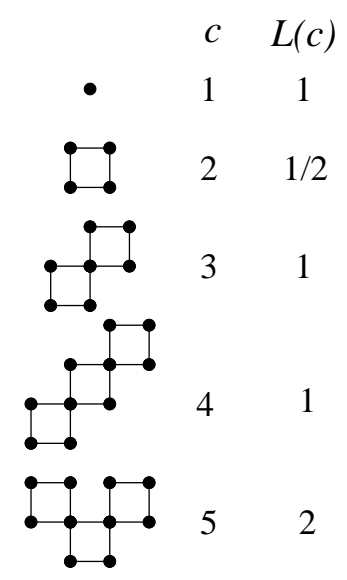

FIG. 3: All topological clusters with up to three squares and their lattice constant for a square expansion.
TABLE III: Number of topological clusters and sum of the lattice constants for clusters with up to five squares in the square lattice. The cluster with zero squares is the single site graph.

\begin{tabular}{crr}
\hline \hline No. of squares No. of topological clusters & $\sum L(c)$ \\
\hline 0 & 1 & 1 \\
1 & 1 & $1 / 2$ \\
2 & 1 & 1 \\
3 & 2 & 3 \\
4 & 5 & $19 / 2$ \\
5 & 11 & $63 / 2$ \\
\hline \hline
\end{tabular}

$60 \mathrm{~h}$ for the bond based NLC expansion (up to 13 bonds).

\section{A. Heisenberg model}

We now consider the antiferromagnetic Heisenberg model (AFHM) on the square lattice. Its Hamiltonian can be written as

$$
\mathcal{H}=\sum_{\langle i, j\rangle} \mathbf{S}_{i} \cdot \mathbf{S}_{j},
$$

where we have chosen the coupling constant to be unity, and the sum runs over nearest-neighbor $(\langle i, j\rangle)$ spins.

The AFHM on the square lattice is known to have an ordered ground state with long-range antiferromagnetic correlations [10]. This model can be efficiently simulated using QMC techniques, such as stochastic series expansions [11]. QMC methods enable one to study much larger system sizes than the ones accessible with exact diagonalization, although the classes of models that can be addressed are limited by the sign problem [12, 13, 14].

We start by studying the temperature dependence of the AFHM energy. In Fig. 4, we show a comparison of the bare sums for the bond, site, and square NLC expansions,

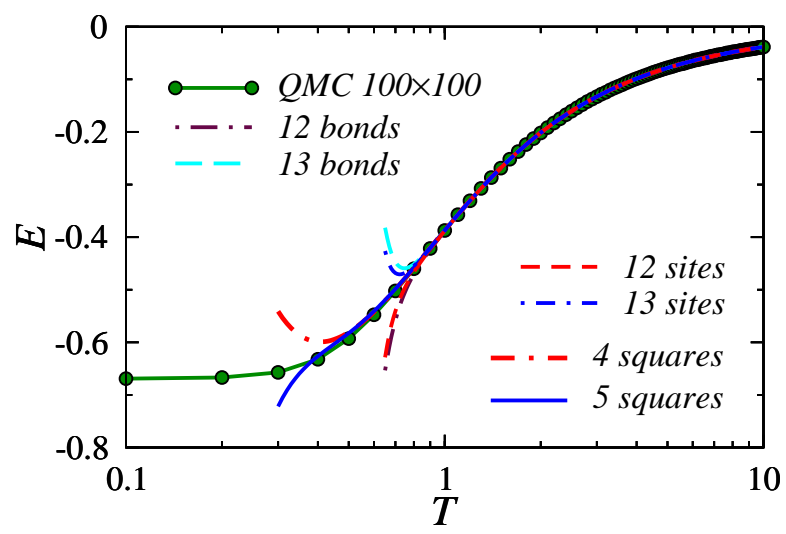

FIG. 4: (Color online) Energy as a function of temperature for the antiferromagnetic Heisenberg model on the square lattice. Bare NLC sums are compared with QMC results for a $100 \times$ 100 lattice. 
with QMC results using the SSE technique [15]. Several issues are apparent in Fig. 4. (i) All NLC expansions give the correct result at high temperatures. (ii) Direct NLC sums can converge below $T=1$, something that is not possible using HTE. (iii) The site expansion, which is closer in spirit to low-temperature expansions, performs better than the bond expansion (closer to HTEs). This occurs even though the site expansion, up to the same order, is less demanding computationally than the bond expansion [there are many topological clusters in the bond expansion (1844) with 13 bonds and 14 sites, while in the site expansion only clusters with up to 13 sites were diagonalized]. (iv) The direct sum of the largest size cluster expansion, the square expansion in this case, converges to the lowest temperature $(T \sim 0.5)$, to be compared with $(T \sim 0.8)$ for the site expansion.

The AFHM on the square lattice is known to develop antiferromagnetic correlations at relatively high temperatures, i.e., it is the kind of model for which the direct NLC sum cannot converge up to very low temperatures. Now, the natural question that arises is how low in temperature can one go by using the sequence extrapolation techniques discussed in Sec. III] In Fig. 5 we show two such extrapolations compared with the QMC results. The subindex following the name of each extrapolation stands for the numbers of cycles of improvements done. In addition, for each cycle of improvement there are, in general, several terms. In what follows, when not explicitly specified otherwise, we will be showing the highest one.

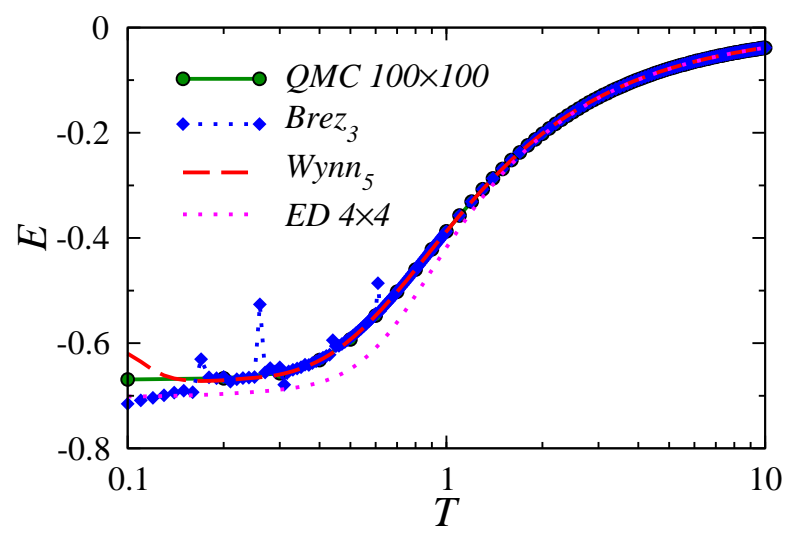

FIG. 5: (Color online) Energy as a function of temperature for the antiferromagnetic Heisenberg model on the square lattice. Different extrapolations for the NLC site expansion are compared with QMC results for a $100 \times 100$ lattice, and with exact diagonalization results for a $4 \times 4$ cluster (with periodic boundary conditions).

Figure 5 shows that extrapolations can indeed work very well within NLC. We only show results for extrapolations of the site expansion since for this expansion we obtain the best results. Already at the level of the bare sum we saw that the site expansion performs better than the bond expansion. On the other hand, the square expansion, which produces the best results for the direct sum, has too few terms to allow for a successful extrapolation scheme to work. [One can also realize that up to 13 sites (with 8739 different topological clusters), the site expansion has explored much more of the lattice than the square expansion (with only 21 different topological clusters).] For the site expansion both Wynn's and Brezinski's algorithms converge, and agree with QMC, down to $T \sim 0.15$. One should notice, however, that while Wynn's results are smooth and on top of the QMC results all the way down to $T \sim 0.15$, some points in the Brezinski's extrapolation fall away from that curve. This is the kind of numerical problem one can run into after several orders of extrapolations. However, with the exception of these few points, Brezinski's results are still well converged and on top of the QMC data. Also shown is the exact diagonalization result for a $4 \times 4$ cluster with periodic boundary conditions. It shows substantial finitesize effects already above $T>1$.

We now consider other thermodynamic quantities of interest, such as the entropy and the specific heat. For the former, we have already shown [5] NLC results for Brezinski's and Wynn's extrapolations compared with the results of Bernu and Misguich [9] (obtained by integrating their specific heat curves). They exhibit a perfect agreement down to $T \sim 0.3$, where $S \sim 0.05$. Here, we will show the results for the specific heat.

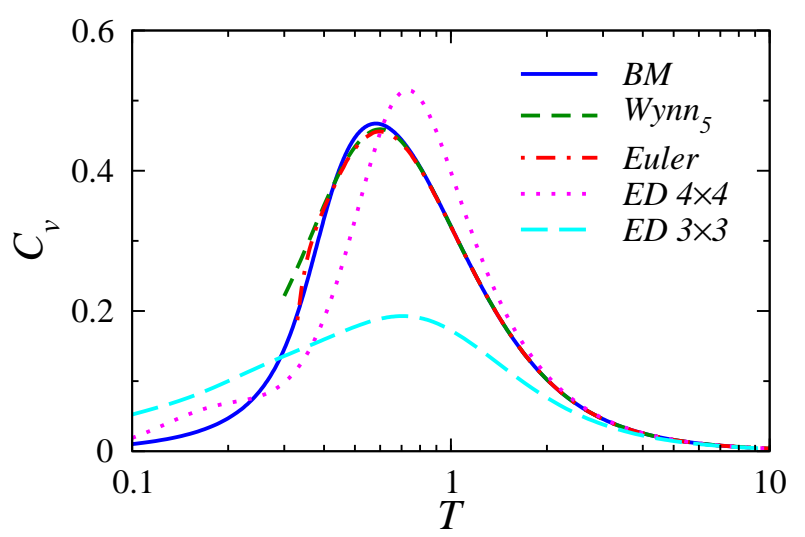

FIG. 6: (Color online) Specific heat as a function of temperature for the antiferromagnetic Heisenberg model on the square lattice. Different extrapolations for the NLC site expansion are compared with the results of Bernu and Misguich [9] and with exact diagonalization results of small clusters (with periodic boundary conditions).

In Fig. 6 we compare NLC results for the specific heat (after extrapolation) with the ones obtained by Bernu and Misguich [9]. Both approaches basically agree in the location of the specific heat peak, although they give slightly different peak values. Since NLC results have not converged fully below the peak, they may lead to the same results as Bernu and Misguich if a few more orders are included. Still, for $C_{v}$, NLC performs much better than direct Pade extrapolation of HTEs. QMC simulations for the specific heat also suffer from large errors ( $C_{v}$ has to be obtained as derivative of the energy), 
and do not allow one to select any of the linked cluster results over the other [15].

We also show in Fig. 6 the specific heat results from the exact diagonalization of $4 \times 4$ and $3 \times 3$ clusters with periodic boundary conditions ( $\mathrm{PBCs}$ ), which helps to give an idea of the order of finite size effects in this model. They lead to a peak in the specific heat that is neither correct in its position nor height. There is another point to consider when comparing NLC with ED especially in dimensions greater than 1 . In the former case, we consider all possible clusters that make the NLC expansion consistent, without any biasing for any type of order. In exact diagonalization studies, PBCs can bias the system towards or away from certain types of order. For example, in the AFHM the $3 \times 3$ cluster with PBCs has much bigger finite-size effects, because PBCs frustrates antiferromagnetism. This issue may become important when the model under consideration has several competing orders, and the selection of a particular finite size cluster may artificially favor one order over the other. NLC, similar to HTE, does not suffer from this problem, and gives an unbiased answer for the thermodynamic properties.

\section{B. $X Y$ model in a staggered transverse field}

As discussed in Ref. [5], NLC is ideal to study models that stay short ranged at all temperatures or models in which correlations build up slowly. In this section we discuss an example of the former case, the $X Y$ model in a staggered transverse field. Its Hamiltonian can be written as

$$
\mathcal{H}=\sum_{\langle i, j\rangle}\left(S_{i}^{x} S_{j}^{x}+S_{i}^{y} S_{j}^{y}\right)+\Delta \sum_{i}(-1)^{i_{x}+i_{y}} S_{i}^{z},
$$

where we have chosen the $X Y$ coupling to be unity, the sum runs over nearest-neighbor $(\langle i, j\rangle)$ spins, and the last

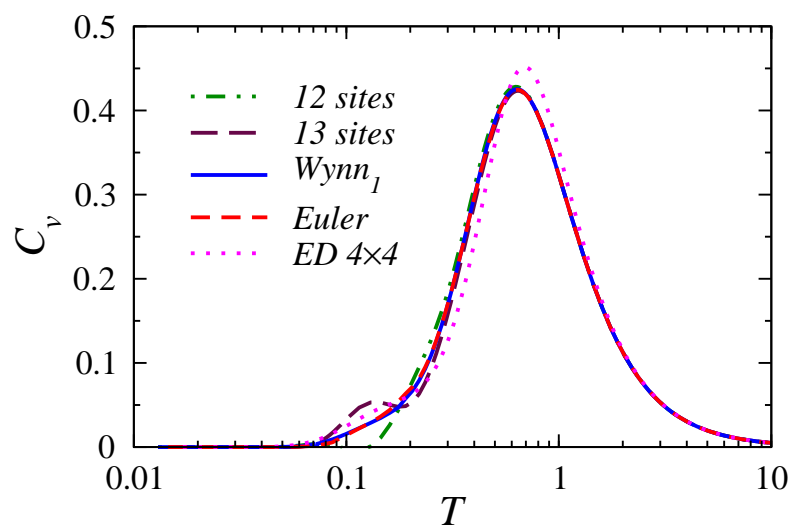

FIG. 7: (Color online) Specific heat as a function of temperature for the $X Y$ model in a staggered transverse field. $\Delta=1$ so that the system is in the insulating regime. Direct sums and different extrapolations for the NLC site expansion are compared with exact diagonalization results for a $4 \times 4$ cluster (with periodic boundary conditions). term describes the staggered field with strength $\Delta$.

The $X Y$ model in a staggered transverse field can be mapped onto a hard-core boson model, at half filling, with a staggered site-dependent chemical potential. Such a model has been recently studied in one [16], two [17], and three dimensions [18]. In 1D, due to its mapping to noninteracting fermions, one can realize that there is, at zero temperature, a phase transition from a superfluid to an insulating phase for $\Delta_{c}=0$, i.e., any finite $\Delta$ produces an insulating phase [16]. In two dimensions this model has been studied by means of QMC simulations, in this case the transition between the superfluid (also Bose-Einstein condensed phase) and the insulating phase occurs, at zero temperature, when $\Delta_{c}=0.992$ [17]. Finally, in three dimensions this model has been used to rigorously prove the existence of Bose-Einstein condensation and Mott insulating phases when tuning the strength of the staggered chemical potential [18].

In what follows we consider the two-dimensional case. In Fig. 7 we show the specific heat as a function of the temperature in a case where the system is insulating at zero temperature. We have chosen $\Delta=1$, close to the critical value $\Delta_{c}$ for the superfluid-insulator transition. Due to the presence of a gap at zero temperature we can be certain that correlations stay short ranged at all temperatures. However, they are not negligible, and the direct sum of the NLC site expansion exhibits a small oscillation below the peak in the specific heat. A fully converged result, at all temperatures, can be obtained after just one cycle of improvement with Wynn's algorithm or using Euler's transformation. Figure 7 also shows that the exact diagonalization results for a $4 \times 4$ cluster with periodic boundary conditions still suffer from apparent finite size effects.

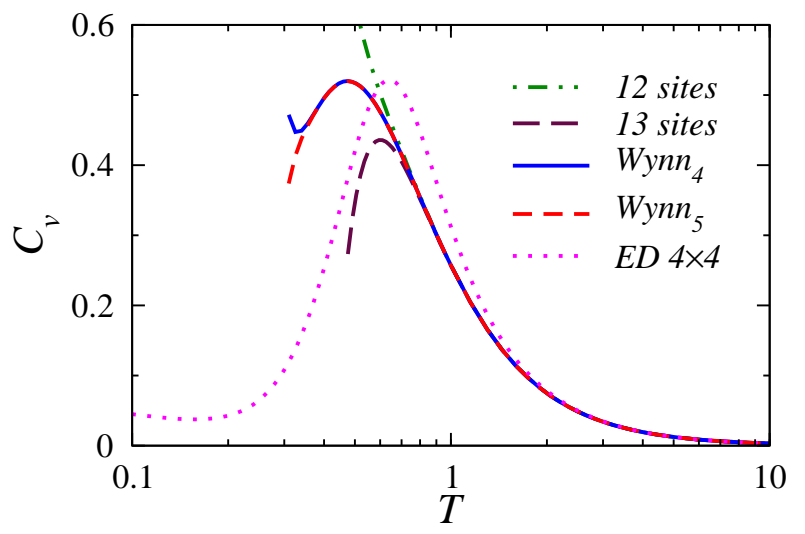

FIG. 8: (Color online) Specific heat as a function of temperature for the $X Y$ model in a staggered transverse field, with $\Delta=0.5$. Direct sums and different extrapolations for the NLC site expansion are compared with exact diagonalization results for a $4 \times 4$ cluster (with periodic boundary conditions).

Once in the regime of $\Delta$ where the system is superfluid at zero temperature, the convergence of the NLC direct sum does not reach very low temperatures, but sequence extrapolations allow one to reach the region below the 
peak in the specific heat. This can be seen in Fig. 8 . As expected, in this case the difference with ED is even larger than when $\Delta>\Delta_{c}$.

\section{Ising model}

To conclude this section on spin models on the square lattice, we discuss in this subsection the Ising model

$$
\mathcal{H}=\sum_{\langle i, j\rangle} S_{i}^{x} S_{j}^{x},
$$

which is an exactly soluble classical model [19].

In two dimensions, the Ising model is known to exhibit a finite-temperature transition between an ordered (gapped) phase, and a disordered high-temperature phase. As shown in Fig. 9, the specific heat exhibits a divergence at the critical point, which is known to be logarithmic [19]. For NLC, the Ising model reduces to a counting problem as the Hamiltonian is already diagonal. In Fig. 9 we show direct sums for the site based expansion up to 15 sites. Surprisingly, Wynn's extrapolations allow one to obtain very good estimates of the specific heat very close to the critical point.

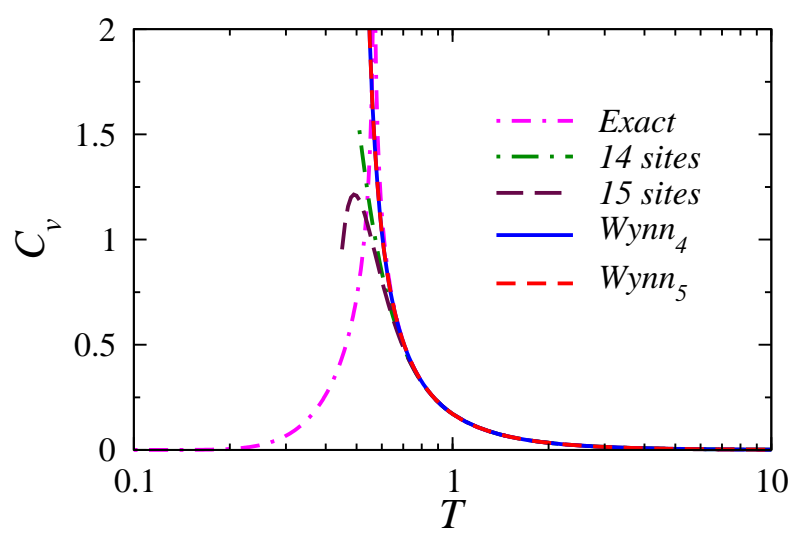

FIG. 9: (Color online) Specific heat as a function of temperature for the Ising model. Direct sums and different extrapolations for the NLC site expansion are compared with exact analytical results.

We should stress, however, that for finite-temperature phase transitions with power-law singularities, HTE is probably the most efficient way to go. This is because such singularities can be built into the extrapolation. We do not know if this is possible within NLC.

\section{TRIANGULAR LATTICE}

In this section we consider the triangular lattice. We discuss three different choices of basic clusters that allow one to build a consistent NLC expansion.

The first possibility is, as in the square lattice, to build all possible clusters with up to a given number of bonds.
TABLE IV: Number of topological clusters and sum of the lattice constants for clusters with up to 12 bonds in the triangular lattice. The cluster with 0 bonds is the one site graph.

\begin{tabular}{crr}
\hline \hline No. of bonds No. of topological clusters & $\sum L(c)$ \\
\hline 0 & 1 & 1 \\
1 & 1 & 3 \\
2 & 1 & 15 \\
3 & 3 & 91 \\
4 & 5 & 603 \\
5 & 12 & 4215 \\
6 & 28 & 30535 \\
7 & 72 & 226905 \\
8 & 198 & 1718454 \\
9 & 590 & 13207569 \\
10 & 1817 & 102707301 \\
11 & 5886 & 806366139 \\
12 & 19753 & 2086381866 \\
\hline \hline
\end{tabular}

Their number of topological clusters, and sum of $L(c)$, when grouped by the number of bonds is presented in Table IV.

From the number of topological clusters and the sum of lattice constants in Table IV one can see that the number of graphs in the triangular lattice grows much faster than in the square lattice. This is because in the triangular lattice each bond has ten nearest-neighbor bonds, as opposed to six in the square lattice. (The number of nearest-neighbor bonds determines the rate of growth of the number of possible clusters.)

A second natural choice is to build all clusters with up to a given number of sites. Once again, when building the Hamiltonian for such clusters one needs to place the maximum number of bonds possible in them. This

TABLE V: Number of topological clusters and sum of the lattice constants for clusters with up to 13 sites in the triangular lattice.

\begin{tabular}{rrr}
\hline \hline No. of sites No. of topological clusters & $\sum L(c)$ \\
\hline 1 & 1 & 1 \\
2 & 1 & 3 \\
3 & 2 & 11 \\
4 & 4 & 44 \\
5 & 8 & 186 \\
6 & 22 & 814 \\
7 & 54 & 3652 \\
8 & 156 & 16689 \\
9 & 457 & 77359 \\
10 & 1424 & 362671 \\
11 & 4505 & 1716033 \\
12 & 14791 & 8182213 \\
13 & 49138 & 39267086 \\
\hline \hline
\end{tabular}


selection of building blocks for the NLC expansion provides a significant reduction in the number of clusters one needs to consider as compared to the bond expansion. (Each site in the triangular lattice has only six nearest neighbor sites.) In addition, having more compact clusters, this expansion performs better and allows for better extrapolations in the intermediate and low-temperature regimes. The number of topological clusters and sum of lattice constants for the site based NLC expansion is shown in Table V]

A triangular lattice is made out of triangles, so it is also possible to develop a NLC for the triangular lattice where the clusters consist of closed triangles. However, in this case, a consistent NLC scheme requires that one restricts the calculation to a single site plus clusters of up (or down) pointing triangles only. The reason for this restriction is that all bonds of the triangular lattice belong to a unique up (or down) pointing triangle. Different triangles should only share sites. The number of topologically distinct clusters with 1 through 8 triangles, and the sum of their lattice constant is shown in Table VI (We have grouped them by the number of triangles.)

TABLE VI: Triangular lattice number of topological clusters and sum of the lattice constants for clusters with up to eight triangles. The cluster with 0 triangles is the single site.

\begin{tabular}{crr}
\hline \hline No. of triangles No. of topological clusters & $\sum L(c)$ \\
\hline 0 & 1 & 1 \\
1 & 1 & $1 / 3$ \\
2 & 1 & 1 \\
3 & 3 & $11 / 3$ \\
4 & 5 & $44 / 3$ \\
5 & 12 & 62 \\
6 & 35 & $814 / 3$ \\
7 & 98 & $3652 / 3$ \\
8 & 299 & 5563 \\
\hline \hline
\end{tabular}

Notice that one advantage of the triangle-based expansion in the triangular lattice, over the square-based expansion in the square lattice, is that in the former the maximum number of lattice sites of a cluster with $N_{t}$ triangles is $2 N_{t}+1$, while in the square lattice it is $3 N_{s}+1$ ( $N_{s}$ being the number of squares). This means that one can fully diagonalize clusters with more triangles than squares, which helps both for the bare NLC sums as well as for extrapolations. In the next subsections we apply the above expansions to Heisenberg and Ising models on the triangular lattice.

\section{A. Heisenberg model}

The triangular-lattice antiferromagnetic Heisenberg model is a fascinating quantum spin model, which has long-range order at $T=0[20]$ but with spin-spin correlations that remain short range down to fairly low tem- peratures [21]. In contrast to the square lattice AFHM, the AFHM on the triangular lattice shows no evidence for a renormalized classical behavior [22, 23, 24] down to lowest temperatures that can be reached in HTE. It is a frustrated spin model so that QMC methods suffer from a sign problem. It has recently been argued [25, 26] that the anomalous finite-temperature behavior is due to the excitation of rotons, which leads to high entropy at relatively low temperatures.

The specific heat of the triangular lattice AFHM is a quantity that could be of direct experimental interest. It has also been calculated from HTE by the recent approach of Bernu and Misguich (BM) [9]. These authors found that direct Pade approximants [21] not only fail at surprisingly high temperatures but are also unable to correctly locate the maximum of the specific heat and its height.

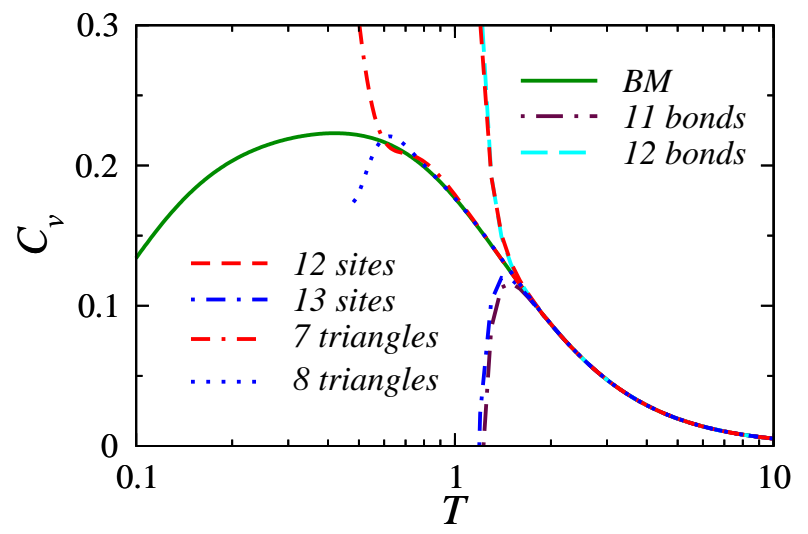

FIG. 10: (Color online) Specific heat as a function of temperature for the Heisenberg model on a triangular lattice. Direct sums are compared with BM results in Ref. [9].

In Fig. 10 we show the bare sums for the three possible NLC expansions discussed before, and compare them with the BM results [9]. The bond and site expansions, up to 12 bonds and 13 sites, respectively, are well converged only at high temperatures (up to $T \sim 2$ ), with the site expansion being slightly better. On the other hand, the triangle based expansion converges down to a lower temperature $T \sim 0.6$. This temperature is very close to the lowest temperature up to which direct Pade extrapolations agree with BM results.

On performing extrapolations over the bare sums shown in Fig. 10, we found that the site expansion is the one that enables an improvement of the convergence to the lowest temperature. (The bare results for the triangle based expansion can hardly be improved by sequence extrapolation methods.) In Fig. 11] we show results for two possible extrapolations of the site based NLC expansion compared with BM results [9].

Notice that we have included two terms for each extrapolation scheme. To understand what they mean one has to realize that up to 13 sites Euler transformation allows for 13 terms, from which we have taken the first four to be the bare sums, and starting with the fifth we 
have applied the transformation as explained in Sec. III Hence, in Fig. 11 we are showing the last two terms (in Sec. IV we showed only the last one). For Wynn's approach on the other hand, one should remember that two terms are lost after each cycle of improvement. So after, five cycles (the case in Fig. 111) ten out of the initial 13 terms in the bare sum are lost, i.e., in Fig. 11 we are showing the last two of the remaining three.

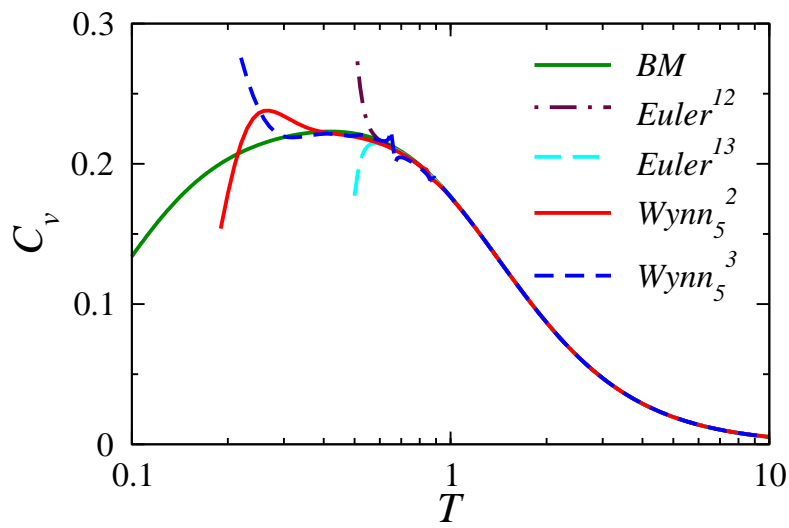

FIG. 11: (Color online) Specific heat as a function of temperature for the Heisenberg model on a triangular lattice. Extrapolations are compared with BM results in Ref. [9]. The superindex refers to the term in the extrapolation (see text for details).

Figure 11]shows that while Euler transformation allows one to extend the convergence of the site based expansion to the region where the triangle based expansion was convergent, Wynn's extrapolation scheme enables one to obtain results at lower temperatures (up to $T \sim 0.3$ ). In contrast to direct Pade for HTE, Wynn's scheme for NLC allows one to reach the maximum of the specific heat as predicted by BM [9].

A second quantity of much experimental interest is the uniform susceptibility 27]. In Fig. 12 we show NLC (bare and extrapolated) results for the uniform susceptibility $(\chi)$ of the AFHM on the triangular lattice. NLC results are compared for this quantity with series extrapolations of HTE obtained by integrated differential approximants [27]. (Notice that the BM approach [9] is not suitable for calculations of $\chi$.)

A comparison between the results for $\chi$ and $C_{v}$ helps to understand why the flexibility one has for selecting different kind of clusters in building the NLC expansion can be useful. In contrast to $C_{v}$, the results of the bare sums for the site based and triangle based NLC expansions for the susceptibility converge well up to about the same high temperature (see Fig. 12 vs Fig. 10). This might suggest that the triangle based expansion may not bring any advantage for this quantity. However, in contrast to $C_{v}$, series extrapolations extend the region of convergence for $\chi$ for the triangle based NLC ( $T$ Wynn in Fig. 12), and allow one to reach lower temperatures than the extrapolations for the site based expansion $(S$ Wynn in Fig. (12). Notice that in the region where NLC

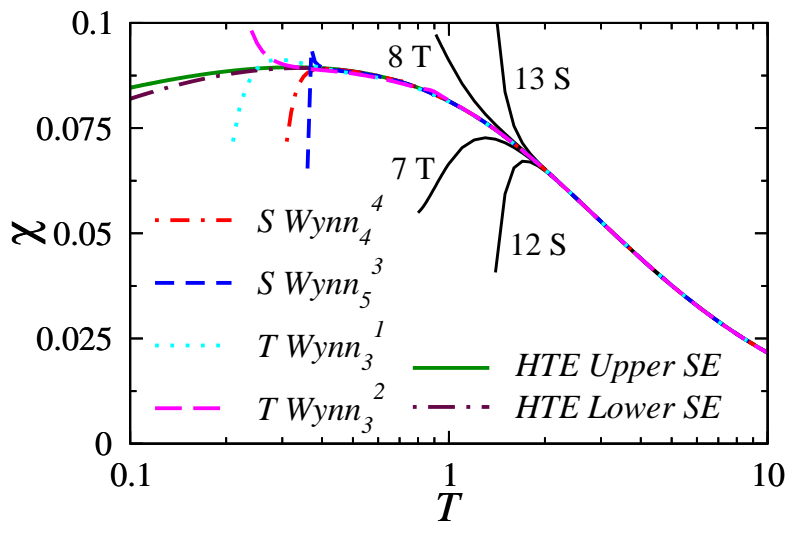

FIG. 12: (Color online) Uniform susceptibility as a function of temperature for the AFHM on a triangular lattice. NLC bare sums are shown for up to 7 and 8 triangles $(7 \mathrm{~T}$ and 8 $\mathrm{T}$ in the figure), and up to 12 and 13 sites (12 $\mathrm{S}$ and $13 \mathrm{~S}$ in the figure). The corresponding extrapolations for the site based ( $S$ Wynn) and triangle based ( $T$ Wynn) expansions are compared with series extrapolation results of HTE, obtained by integrated differential approximants [27]. For the latter, only the upper and lower boundaries are shown.

extrapolations are well converged they are in excellent agreement with extrapolations of HTE obtained by integrated differential approximants [27]. For $\chi$, the integrated differential approximants of HTE (which do not work so well for $C_{v}$ ) appear to have convergence to lower temperatures than the ones reached with NLC.

\section{B. Ising model}

The Ising model [Eq. (14)] on the triangular lattice is an exactly soluble model [28, 29, 30]. At zero temperature it exhibits power-law decaying spin correlations and an extensive entropy $S=0.3231$.

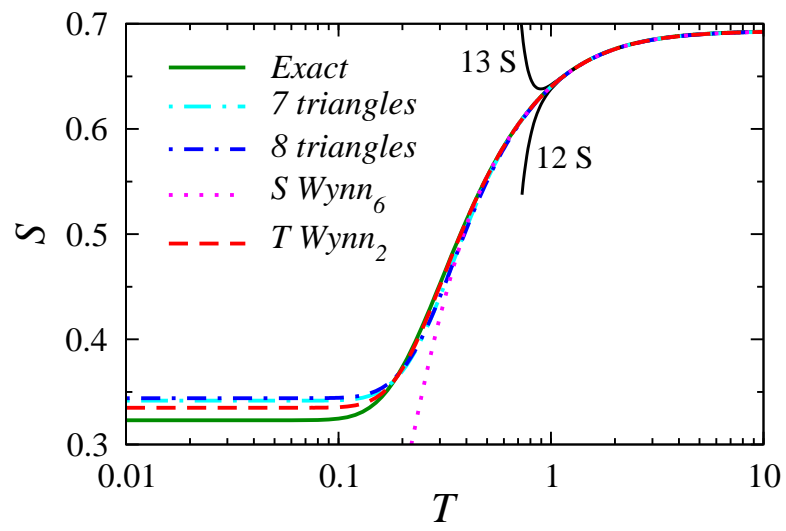

FIG. 13: (Color online) Entropy as a function of temperature for the Ising model on a triangular lattice. NLC bare sums are shown for up to 7 and 8 triangles, and up to 12 and 13 sites (12 $\mathrm{S}$ and $13 \mathrm{~S}$ in the figure). The corresponding extrapolations for the site based (S Wynn) and triangle based ( $\mathrm{T}$ Wynn) expansions are compared with the exact analytical result. 
For this model, the triangle based NLC expansion converges down to low enough temperatures that it even allows one to study some ground state properties, for example, the entropy shown in Fig. 13. One can see that the site based expansion converges only up to $T \sim 1$. Hence, the triangle based expansion provides one with a qualitative improvement over site (and bond) expansion. Adding up contributions from clusters up to $N_{t}=1,2$, $3,4,5,6,7,8$ triangles leads to estimates of zero temperature entropy of $S=0.6931,0.4055,0.3677,0.3677$, $0.3499,0.3521,0.3417,0.3440$, respectively. In this case, the convergence to the thermodynamic limit result is power law in $1 / N_{t}$, compared to an exponential convergence in the kagomé case [5], which is not surprising given that the triangular-lattice model is critical [30].

Wynn's extrapolation of the triangle expansion, up to 8 triangles, improves towards the thermodynamic limit result as shown in Fig. 13. At low temperatures it gives an estimate of the entropy $S=0.3349$. The extrapolation for the site expansion, on the other hand, only converges down to $T \sim 0.3$.

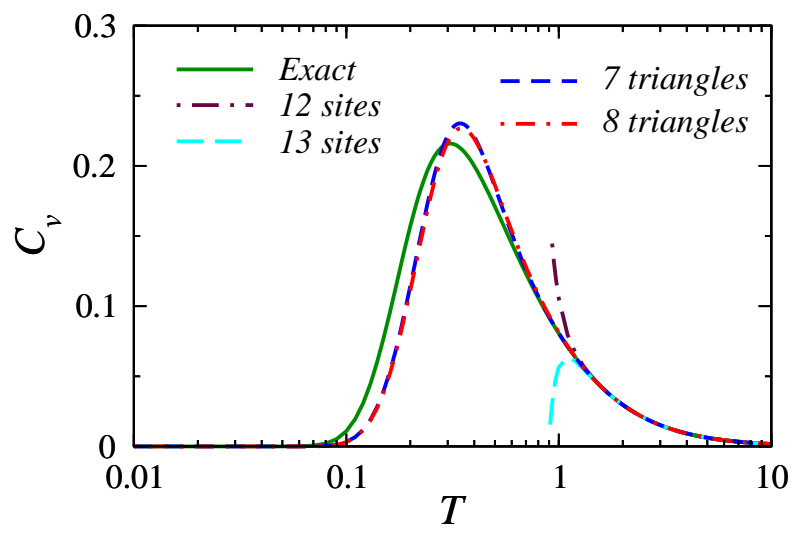

FIG. 14: (Color online) Specific heat as a function of temperature for the Ising model on a triangular lattice. NLC bare sums are shown for up to 7 and 8 triangles, up to 12 and 13 sites, and compared with the exact analytical result.

A comparison of the NLC results for the specific heat with the exact analytical calculation is shown in Fig. 14. For this quantity, extrapolations of the site, bond, and triangle based expansions do not allow one to improve over the direct triangle based sum, so we do not show them there. It is remarkable, however, that even though the system is critical the results of the triangle based bare sums are not far from that exact result.

\section{KAGOMÉ LATTICE}

In this section we consider the kagomé lattice. As before, we discuss three different choices of basic clusters that allow one to build a consistent NLC expansion.

The first choice, again, is to use all clusters up to a given number of bonds. The number of topological clus-
TABLE VII: Number of topological clusters and sum of the lattice constants for clusters with up to 13 bonds in the kagomé lattice. The cluster with 0 bonds is the one site graph.

\begin{tabular}{crr}
\hline \hline No. of bonds No. of topological clusters & $\sum L(c)$ \\
\hline 0 & 1 & 1 \\
1 & 1 & 2 \\
2 & 1 & 6 \\
3 & 3 & $62 / 3$ \\
4 & 4 & 77 \\
5 & 8 & 304 \\
6 & 17 & $3752 / 3$ \\
7 & 36 & 5294 \\
8 & 81 & 22845 \\
9 & 194 & $299924 / 3$ \\
10 & 481 & 442507 \\
11 & 1235 & 1977572 \\
12 & 3297 & $26729935 / 3$ \\
13 & 8944 & 40418174 \\
\hline \hline
\end{tabular}

ters, and sum of $L(c)$, when grouped by their number of bonds is presented in Table. VII]

A second choice is to build all clusters with up to a given number of sites. When building the Hamiltonian for such clusters one needs to place the maximum number of bonds possible in them. The number of topologies and sum of lattice constants for the site based NLC expansion is shown in Table VIII. We have grouped them by number the number of sites.

TABLE VIII: Number of topological clusters and sum of the lattice constants for clusters with up to 15 sites in the kagomé lattice.

\begin{tabular}{rrr}
\hline \hline No. of sites No. of topological clusters & $\sum L(c)$ \\
\hline 1 & 1 & 1 \\
2 & 1 & 2 \\
3 & 2 & $14 / 3$ \\
4 & 2 & 12 \\
5 & 4 & 33 \\
6 & 7 & $281 / 3$ \\
7 & 12 & 272 \\
8 & 22 & 805 \\
9 & 45 & 2420 \\
10 & 88 & 7358 \\
11 & 183 & 22581 \\
12 & 389 & $209552 / 3$ \\
13 & 842 & 217522 \\
14 & 1855 & 681224 \\
15 & 4162 & 2143905 \\
\hline \hline
\end{tabular}

Since the kagomé lattice consists of corner sharing triangles, the triangle-based NLC, in this case, involves all elementary triangles. This selection of building blocks for 
NLC reduces dramatically the number of clusters to be considered. The number of topologically distinct clusters with 1 through 8 triangles, and the sum of their lattice constant is shown in Table IX]. (We have grouped them by the number of triangles.)

TABLE IX: Kagomé lattice number of topological clusters and sum of the lattice constants for clusters with up to eight triangles. The cluster with 0 triangles is the single site.

\begin{tabular}{crr}
\hline \hline No. of triangles No. of topological clusters & $\sum L(c)$ \\
\hline 0 & 1 & 1 \\
1 & 1 & $2 / 3$ \\
2 & 1 & 1 \\
3 & 1 & 2 \\
4 & 2 & $14 / 3$ \\
5 & 2 & 12 \\
6 & 5 & $94 / 3$ \\
7 & 7 & $250 / 3$ \\
8 & 15 & 225 \\
\hline \hline
\end{tabular}

In Ref. [5] we have already discussed extensively several spin models on the kagomé lattice. Hence, here we will restrict our analysis to the specific heat and the uniform susceptibility of the AFHM.

\section{A. Heisenberg model}

The kagomé-lattice AFHM model has been argued to have short-ranged spin-spin correlations down to $T=0$ [31, 32, 33]. Its thermodynamic properties have also been of considerable interest [34]. In particular, an issue that is still under debate (motivated by experiments on $\mathrm{He}^{3}$ on graphite) is whether this model exhibits a two-peaked structure in the specific heat [35, 36].

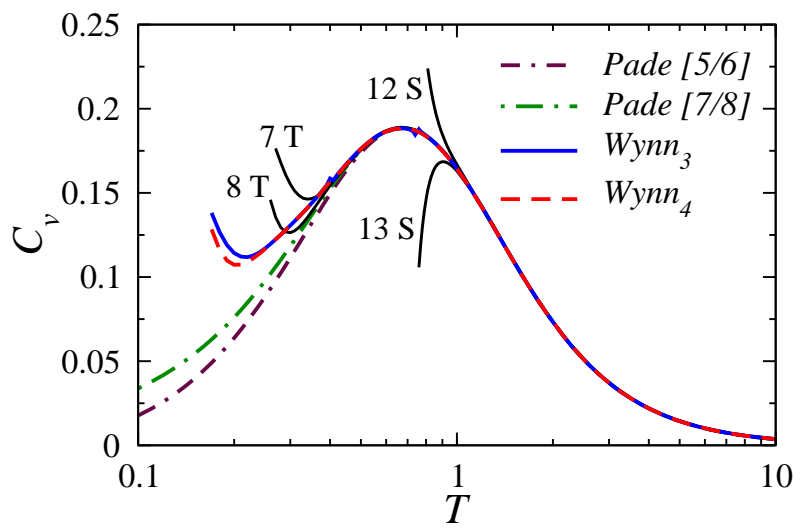

FIG. 15: (Color online) Specific heat as a function of temperature for the Heisenberg model on a kagomé lattice. Direct sums, for up to 7 and 8 triangles ( $7 \mathrm{~T}$ and $8 \mathrm{~T}$ in the figure) and up to 12 and 13 sites (12 $\mathrm{S}$ and $13 \mathrm{~S}$ in the figure), are compared with extrapolations for the triangle expansion and with two Pade approximants from Ref. 36].
In Ref. [5] we have already studied the specific heat of the AFHM. There we compared the direct sums of the triangle expansion with Pade approximants from HTE [36], which showed an overall good agreement down to $T \sim 0.3$. The triangle expansion for the specific heat on the kagomé lattice, in contrast to the triangular lattice in the previous section, allows for an acceleration of the convergence by means of Wynn's extrapolations.

In Fig. 15, we compare the bare sums for the site and triangle expansions with results of Wynn's extrapolation and Pade approximants [36]. As seen in Fig. 15, the results for Wynn's extrapolation appear to converge down to $T \sim 0.2$ and exhibit a clear deviation from the Pade results. The deviations are, one could say, in the right direction since the extrapolation of the specific-heat HTE down to $T=0$ has a large missing entropy [36].

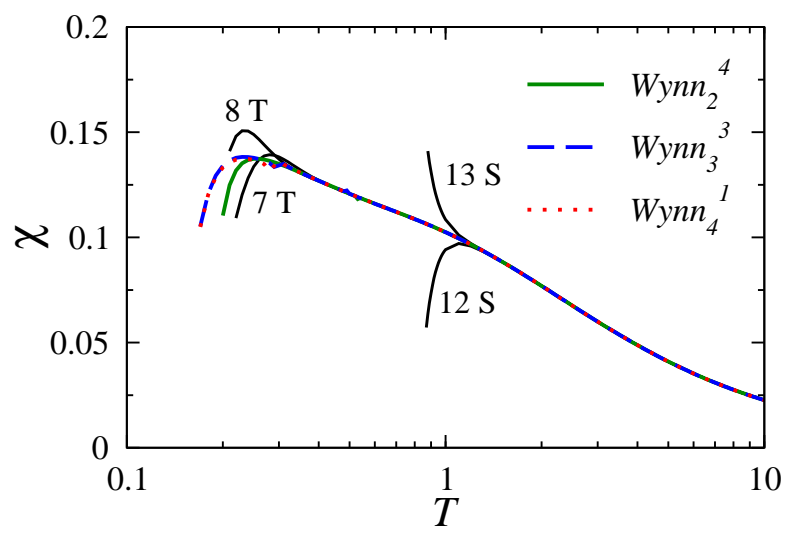

FIG. 16: (Color online) Uniform susceptibility as a function of temperature for the AFHM on a kagomé lattice. NLC bare sums are shown for up to 7 and 8 triangles $(7 \mathrm{~T}$ and $8 \mathrm{~T}$ in the figure), and up to 12 and 13 sites (12 $\mathrm{S}$ and $13 \mathrm{~S}$ in the figure). The corresponding Wynn's extrapolations for triangle based expansions are also shown.

To conclude this section we show in Fig. 16 NLC results for the bare and extrapolated sums of the uniform susceptibility $(\chi)$ of the AFHM on the kagomé lattice. Similar to the extrapolations for the specific heat, Wynn's extrapolations are well converged down to $T \sim 0.2$, while the direct sums for the triangle expansion converge down to $T \sim 0.3$. On the other hand, the site based expansion, up to 13 sites, converges only down to $T \sim 1$, which is the same temperature one can access with HTE without extrapolations. Overall, for the kagomé lattice we have found that direct and extrapolated sums of the triangle based expansion converge better for the thermodynamic observables considered (energy, entropy, specific heat, and uniform susceptibility) than the site and bond based expansions.

\section{CONCLUSIONS}

We have presented an extensive discussion of the numerical linked cluster algorithm introduced in Ref. 
[5]. We have detailed how to construct NLC starting from different building blocks on square, triangular, and kagomé lattices. Several sequence extrapolation techniques, which we have used to accelerate NLC convergence, have also been discussed.

In order to show how NLC works for models with different ground states and orders, we have studied several spin models on square, triangular, and kagomé lattices. We have shown that NLC is better suited for systems that remain short ranged at all temperatures (such as the $X Y$ model in a staggered field), and for models where correlations build up slowly so that they become large only at very low temperatures. A good example of the latter case is the AFHM on the kagomé lattice, for which well converged results could be obtained down to $T \sim 0.3$ without the need of using sequence extrapolation techniques.

Similar to HTE, NLC also allows for extrapolations beyond the region of convergence provided by clusters up to a given system size. It is important to note that within NLC the region of convergence is dictated by the largest cluster sizes considered, and by the range of correlations in the thermodynamic system. Hence, even without extrapolations, one can, in principle, extend the region of convergence by including larger clusters. In this respect NLC is fundamentally different from HTE, whose region of convergence is dictated by the dominant microscopic energy scale, and including larger clusters can only help with extrapolations as they do not improve the convergence of the direct sum. Extrapolations within NLC al- low one to go to temperatures lower than accessible by means of direct Pade approximants for HTE. Examples where NLC is superior in this respect include the specific heat in the triangular and kagomé lattices.

Finally, we have also compared NLC results with those obtained from exact diagonalization of clusters with periodic boundary conditions. We have shown that NLC provides accurate results even where ED still suffers from very large finite size effects. Even for short ranged models such as the $X Y$ model in a staggered field, ED can fail to predict, for example, the position and height of the peak in the specific heat.

Although it was not implemented here, one way to improve NLC convergence at lower temperatures would be to use Lanczos type methods to diagonalize larger clusters. Larger clusters would become possible if one was to focus only on low lying states rather than the full diagonalization that we have used in this work.

\section{Acknowledgments}

This work was supported by the US National Science Foundation, Grant Nos. DMR-0240918, DMR-0312261, and PHY-0301052. We are grateful to B. Bernu and G. Misguich for providing us with their data from Ref. [9], and to $\mathrm{R}$. Yu for providing us with the QMC results presented in Sec. IV
[1] J. Jaklic and P. Prelovsek, Adv. Phys. 49, 1 (2000).

[2] A. W. Sandvik, Phys. Rev. B 59, R14157 (1999).

[3] For a general introduction see C. Domb and M. S. Green, Phase Transitions and Critical Phenomena, (Academic Press, New York, 1974), Vol. 3.

[4] A. J. Guttmann, in Phase Transitions and Critical Phenomena, edited by C. Domb and J. Lebowitz (Academic Press, London, 1989), Vol. 13.

[5] M. Rigol, T. Bryant, and R. R. P. Singh, Phys. Rev. Lett. 97, 187202 (2006).

[6] A similar idea for ground state properties was considered earlier by A. C. Irving and C. J. Hamer, Nucl. Phys. B 230, 361 (1984).

[7] W. H. Press, B. P. Flannery, S. A. Teukolsky, and W. T. Vetterling, Numerical Recipes in Fortran (Cambridge University Press, Cambridge, England, 1999), Sec. 5.1.

[8] J. Oitmaa, C. Hamer, and W-H. Zheng, Series Expansion Methods for Strongly Interacting Lattice Models (Cambridge University Press, Cambridge, 2006).

[9] B. Bernu and G. Misguich, Phys. Rev. B 63, 134409 (2001).

[10] R. R. P. Singh, Phys. Rev. B 39, 9760 (1989); J.-K. Kim and M. Troyer, Phys. Rev. Lett. 80, 2705 (1998).

[11] A. W. Sandvik and J. Kurkijarvi, Phys. Rev. B 43, 5950 (1991); A. W. Sandvik, J. Phys. A 25, 3667 (1992); Phys. Rev. B 59, R14157 (1997).

[12] E. Y. Loh, Jr., J. E. Gubernatis, R. T. Scalettar, S. R. White, D. J. Scalapino, and R. L. Sugar, Phys. Rev. B
41, 9301 (1990).

[13] P. Henelius and A. W. Sandvik, Phys. Rev. B 62, 1102 (2000).

[14] M. Troyer and U.-J. Wiese, Phys. Rev. Lett. 94, 170201 (2005)

[15] R. Yu (private communication).

[16] V. G. Rousseau, D. P. Arovas, M. Rigol, F. Hébert, G. G. Batrouni, and R. T. Scalettar, Phys. Rev. B 73, 174516 (2006).

[17] A. Priyadarshee, S. Chandrasekharan, J.-W. Lee, and H. U. Baranger, Phys. Rev. Lett. 97, 115703 (2006).

[18] M. Aizenman, E. H. Lieb, R. Seiringer, J. P. Solovej, and J. Yngvason, Phys. Rev. A 70, 023612 (2004).

[19] L. Onsager, Phys. Rev. 65, 117 (1944).

[20] B. Bernu, C. Lhuillier, and L. Pierre, Phys. Rev. Lett. 69, 2590 (1992).

[21] N. Elstner, R. R. P. Singh and A. P. Young, Phys. Rev. Lett. 71, 1629 (1993); J. Appl. Phys. 75, 5943 (1994).

[22] S. Chakravarty, B. I. Halperin, and D. R. Nelson, Phys. Rev. Lett. 60, 1057 (1988).

[23] P. Azaria, B. Delamotte, and D. Mouhanna, Phys. Rev. Lett. 68, 1762 (1992).

[24] A. V. Chubukov, T. Senthil, and S. Sachdev, Phys. Rev. Lett. 72, 2089 (1994).

[25] W. Zheng, J. O. Fjaerestad, R. R. P. Singh, R. H. McKenzie, and R. Coldea, Phys. Rev. Lett. 96, 057201 (2006); Phys. Rev. B 74, 224420 (2006).

[26] O. A. Starykh, A. V. Chubukov, and A. G. Abanov, Phys. 
Rev. B 74, 180403(R) (2006).

[27] W. Zheng, R. R. P. Singh, R. H. McKenzie, and R. Coldea, Phys. Rev. B 71, 134422 (2005).

[28] G. H. Wannier, Phys. Rev. 79, 357 (1950).

[29] R. M. F. Houtappel, Physica (Amsterdan) 16, 425 (1950).

[30] J. Stephenson, J. Math. Phys. 5, 1009 (1964); 11, 413 (1970).

[31] P. Lecheminant, B. Bernu, C. Lhuillier, L. Pierre, and P. Sindzingre, Phys. Rev. B 56, 2521 (1997).

[32] C. Zeng and V. Elser, Phys. Rev. B 51, 8318 (1995).

[33] R. R. P. Singh and D. A. Huse, Phys. Rev. Lett. 68, 1766 (1992).
[34] P. Sindzingre, G. Misguich, C. Lhuillier, B. Bernu, L. Pierre, Ch. Waldtmann, and H.-U. Everts, Phys. Rev. Lett. 84, 2953 (2000)

[35] V. Elser, Phys. Rev. Lett. 62, 2405 (1989).

[36] N. Elstner and A. P. Young, Phys. Rev. B 50, 6871 (1994).

[37] Notice, that from the expression given in Ref. [4] we have corrected a sign in a subindex.

[38] This limitation, however, can be mitigated by the fact that one can trivially parallelize the NLC calculation. Still, the number of clusters increases exponentially so that only few orders can be gained by parallelization. 